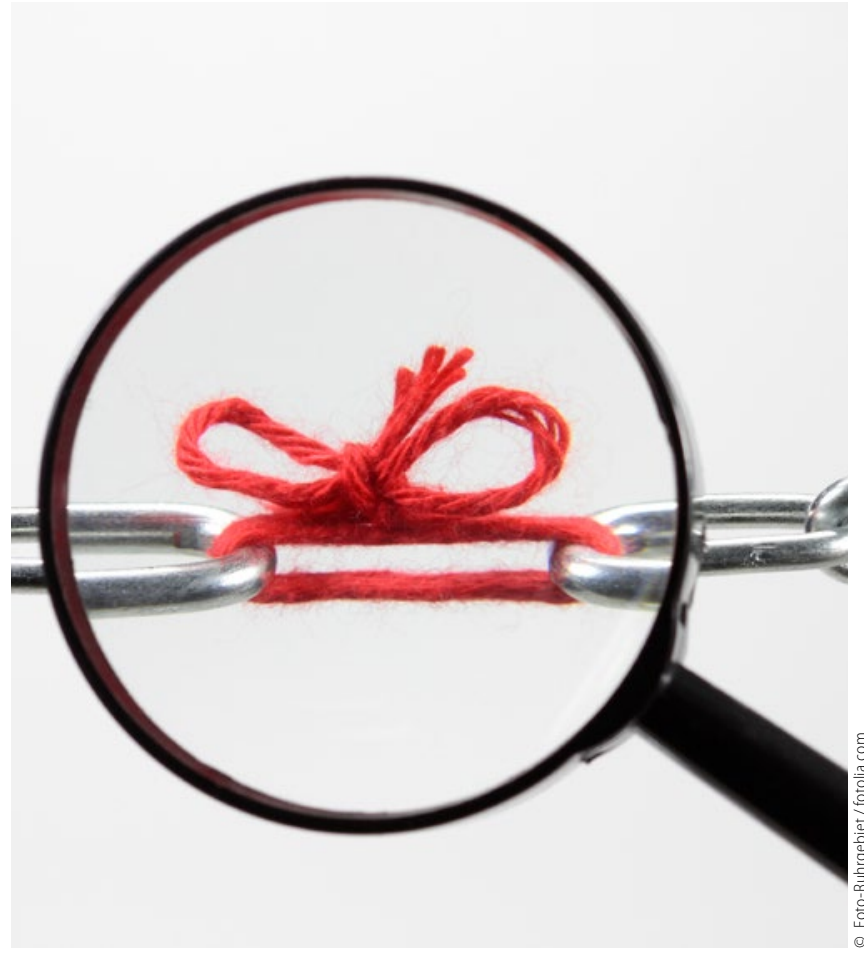

\section{Mogelpackung: Selbstverwaltungsstärkungsgesetz}

Als im Sommer 2016 die ersten Eckpunkte des so genannten Selbstverwaltungsstärkungsgesetzes bekannt wurden, konnte niemand so richtig glauben, dass das Bundesgesundheitsministerium (BMG) mit dieser Sache ernst machen würde. $\mathrm{Zu}$ absurd erschien es, nach den Verfehlungen und Skandalen, die sich in der Kassenärztlichen Bundesvereinigung abgespielt hatten, mit einem Rundumschlag alle Organe der Selbstverwaltung über einen Kamm zu scheren und Regelungen wie auch aufsichtsrechtliche Bestimmungen für alle zu verschärfen. Doch das BMG tat genau dies. Ende September wurde ein Referentenentwurf aus dem Hause von Bundesgesundheitsminister Hermann Gröhe öffentlich, der es in sich hatte. Kurz gesagt, würde die Rechtsaufsicht des BMG über die Selbstverwaltung zur Fachaufsicht mutieren, wenn dieses Vorhaben umgesetzt werden würde. Verschont wurde niemand, selbst der Gemeinsame Bundesausschuss (G-BA), als unabhängiges Gremium, in dem die Entscheidungen über die Leistungen der gesetzlichen Krankenkasse getroffen werden, war im Referentenentwurf von den staatlichen Aufsichtsplänen und Einflussnahme des Ministeriums betroffen. Die Organisationen der Selbstverwaltung und die Verbände liefen Sturm gegen den Referentenentwurf. Von Entmachtung und Ermächtigung war die Rede. Auf höchster Ebene liefen sich die Chefs der Gremien warm zum Protest, um dann in der Anhörung des Ministeriums sachlich, aber pointiert die Argumente vorzutragen. Im BMG wurde klar: Der Entwurf muss überarbeitet werden. Mitte November kam ein neuer - entschärfter - Entwurf ins Kabinett. Ende gut, alles gut? Wohl nicht. Viele Kritikpunkte bleiben. Und so wird dieses Selbstverwaltungsstärkungsgesetz, dass voraussichtlich frühestens im Januar endgültig verabschiedet wird, wohl als das umstrittenste Gesetz aus dem Hause Gröhe in die Geschichte der Legislaturperiode eingehen.

\section{Unsinn: Zahnärztliche MVZ gefährden Versorgung}

Das GKV-Versorgungsstärkungsgesetz (GKV-VSG) hat möglich gemacht, wovon Politiker aus den linkeren Szenen schon lange träumen: Seit Juli 2015 dürfen auch arztgruppengleiche Medizinische Versorgungszentren (MVZ) gegründet werden. Zahnärztlichen MVZ steht also nichts mehr im Weg. Und sie werden auch gegründet. Bis vor zwei Monaten zählte die Kassenzahnärztliche Bundesvereinigung (KZBV) 185 solcher zahnmedizinischer Großpraxen. Der Haken: Die MVZ entstanden vorwiegend in Gebieten, wo sie eigentlich gar nicht gebraucht werden, weil dort der Versorgungsgrad ohnehin hoch ist. Außerdem entschieden sich die MVZ-Betreiber vorrangig für Standorte, an denen Menschen aus hohen Einkommensgruppen leben.

Damit erweist sich das vorgebliche Ziel der Bundesregierung, mit dem Gesetz die flächendeckende ambulante medizinische Versorgung vor allem im ländlichen Raum zu sichern, als fadenscheinig. Diese Fehlentwicklung hält die Zahnärzteschaft für schlichtweg irrsinnig und fordert den Gesetzgeber auf, die Entscheidung, dass arztgruppengleiche MVZ möglich sind, rückgängig zu machen. Die Hauptversammlung des Freien Verbandes Deutscher Zahnärzte (FVDZ) hat einen entsprechenden einstimmigen Beschluss gefasst. Und die Körperschaften sind nicht weniger kritisch: „Mit der Einführung der arztgruppengleichen MVZ im vertragszahnärztlichen Bereich setzt man die wohnortnahe Sicherstellung ohne Not aufs Spiel und fördert stattdessen Großversorgungsstrukturen, Kettennetze und die zunehmende Ökonomisierung und Industrialisierung eines Heilberufs", moniert etwa der KZBV-Vorstandsvorsitzende Dr. Wolfgang Eßer. Mit rein zahnärztlichen MVZ ohne Zulassungsbeschränkung für angestellte Zahnärzte habe der Gesetzgeber eine Kooperationsform zwischen Zahnärzten ermöglicht, die keinen Zusatznutzen für die Versorgung erkennen lasse und die traditionellen Praxisformen erheblich benachteilige. Der Präsident der Bundeszahnärztekammer Dr. Peter Engel bezeichnet MVZ sogar als „Weg in die Discount-Medizin, der unserem freiberuflichen Denken widerspricht und den Patienten schadet“.

FVDZ-Bundesvorsitzender Harald Schrader setzt auf neue Strukturen: „Statt wettbewerbsverzerrender MVZ müssen wir alternative Praxismodelle anbieten, die attraktiver sind als Medizinische Versorgungszentren“, konstatiert er. Die Alternativen müssen so aussehen, dass die Verantwortung für die Behandlung auf jeden Fall bei den Zahnärzten bleibe. Ein Beispiel sei die Flexibilisierung von Berufsausübungsgemeinschaften.

$m f$

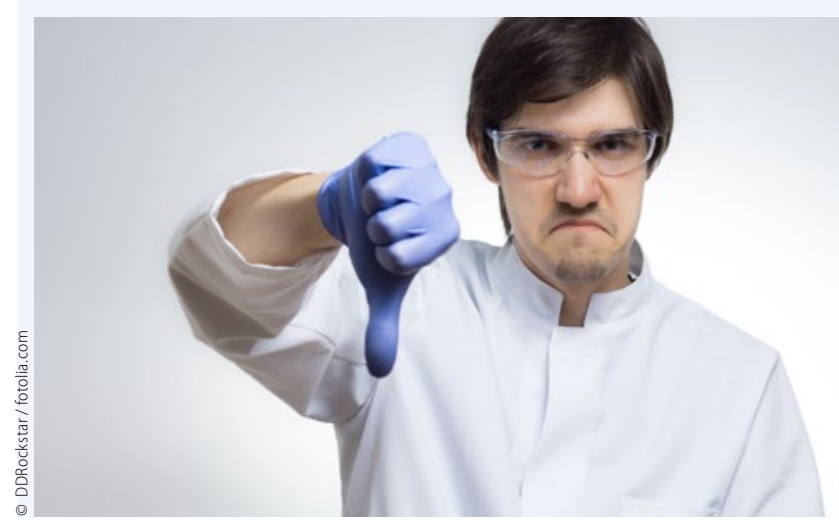

